\title{
Prevalence and incidence of, and risk factors for chronic cough in the adult population: the Rotterdam Study
}

\author{
Johnmary T. Arinze (10), Emmely W. de Roos (10) ${ }^{2,3}$, Leila Karimi $\mathbb{1}^{1}$, \\ Katia M.C. Verhamme (10) ${ }^{1}$, Bruno H. Stricker (10) ${ }^{2}$ and Guy G. Brusselle (10) ${ }^{2,3,4}$
}

Affiliations: ${ }^{1}$ Dept of Medical Informatics, Erasmus University Medical Center, Rotterdam, The Netherlands. ${ }^{2}$ Dept of Epidemiology, Erasmus Medical Center, Rotterdam, The Netherlands. ${ }^{3}$ Dept of Respiratory Medicine, Ghent University Hospital, Ghent, Belgium. ${ }^{4}$ Dept of Respiratory Medicine, Erasmus Medical Center, Rotterdam, The Netherlands.

Correspondence: Guy G. Brusselle. Dept of Respiratory Medicine, Ghent University Hospital, C. Heymanslaan 10, B-9000, Gent, Blegium. E-mail: guy.brusselleduzgent.be

ABSTRACT Chronic cough is a common complaint in the general population but there are no precise data on the incidence of, and prospectively examined risk factors for chronic cough in a population-based setting. Therefore, we investigated the period prevalence, incidence and risk factors for chronic cough in adult subjects.

In a prospective population-based cohort study among subjects aged $\geqslant 45$ years, data on chronic cough were collected on two separate occasions using a standardised questionnaire. Chronic cough was defined as daily coughing for at least 3 months duration during the preceding 2 years. Potential risk factors were gathered by interview, physical examination and several investigations.

Of the 9824 participants in this study, 1073 (10.9\%) subjects had chronic cough at baseline. The prevalence of chronic cough increased with age and peaked in the eighth decade. In subjects aged $<70$ years, chronic cough was more common in women. During an average follow-up of 6 years, 439 incident cases of chronic cough occurred with an overall incidence rate of 11.6 per 1000 person-years (95\% CI 10.6-12.8). In current smokers, the incidence of chronic cough was higher in men. In the multivariable analysis, current smoking, gastro-oesophageal reflux disease (GORD), asthma and COPD were identified as risk factors for chronic cough.

Chronic cough is common among adults and highly prevalent in the older population. Current smoking, GORD, asthma and COPD are independent risk factors for chronic cough. Individuals at risk of developing chronic cough may benefit from smoking cessation and control of the underlying disease.

$@$ ERSpublications

Smokers and individuals with gastro-oesophageal reflux disease, asthma or COPD have an independent risk of developing chronic cough http://bit.ly/31RKk7t

Cite this article as: Arinze JT, de Roos EW, Karimi L, et al. Prevalence and incidence of, and risk factors for chronic cough in the adult population: the Rotterdam Study. ERJ Open Res 2020; 6: 00300-2019 [https://doi.org/10.1183/23120541.00300-2019].

Copyright $\odot$ ERS 2020. This article is open access and distributed under the terms of the Creative Commons Attribution Non-Commercial Licence 4.0. 


\section{Introduction}

Chronic cough is a common complaint in the general population and also one of the most common conditions for which medical care is sought [1]. Globally, it affects approximately $10 \%$ of the adult population and is associated with impaired quality of life $[2,3]$. The diagnosis and treatment of chronic cough are challenging due to the multifactorial nature of its aetiologies $[4,5]$.

Most cases of chronic cough, often defined as cough lasting for $\geqslant 3$ months [6], can be explained by common respiratory and non-respiratory disease conditions such as chronic rhinosinusitis, asthma, chronic obstructive pulmonary disease (COPD), chronic bronchitis, obesity, gastro-oesophageal reflux disease (GORD), lung cancer, heart failure, medications (e.g. angiotensin converting enzyme inhibitors) and other important risk factors, such as smoking, occupational and environmental factors [7-9]. However, some patients with chronic cough do not have any identifiable underlying cause which is consequently called idiopathic chronic cough [10]. In Europe, about half of the patients with chronic cough do not have any known cause [11]. In the light of this evidence, chronic cough has been described as a distinct clinical syndrome, that is, the cough hypersensitivity syndrome [12-14].

The prevalence of chronic cough differs with age, sex and geographical location. It is more common in females and in the elderly population [15]. There are geographical differences in the prevalence of chronic cough with an estimated prevalence of $18.1 \%$ in Oceania, $12.7 \%$ in Europe, $11.0 \%$ in America, $4.4 \%$ in Asia and $2.3 \%$ in Africa $[2,16]$.

Overall, the high prevalence of chronic cough, its negative impact on daily life, diagnostic challenges and limited therapeutic options represent a knowledge gap and obvious need for more research. Apparently, the prevalence of chronic cough has been varyingly reported and there are no precise and prospectively analysed data on the incidence of, and risk factors for chronic cough in the adult population under everyday circumstances [4].

Therefore, the objective of this study was to investigate the period prevalence, incidence and risk factors of chronic cough in a large prospective population-based cohort with long-term follow-up.

\section{Methods}

\section{Study design}

The present study was conducted within the Rotterdam Study, an ongoing prospective population-based cohort study that investigates the occurrence and determinants of chronic diseases in middle-aged and older adults. The details and updates of the study objectives and methods have been previously reported $[17,18]$. In summary, the Rotterdam Study (RS) has approximately 15000 participants, aged $\geqslant 45$ years, enrolled in three cycles (RS I, RS II, and RS III) from the Ommoord district, a well-defined suburb of the city of Rotterdam, the Netherlands. Participants make follow-up visits to a specially built research centre in the district every 4-5 years for specific examinations. Data about life-style and medical history were collected during home interview by trained research assistants. In addition, other relevant data were retrieved from medical records of the general practitioners (GPs), nursing homes, and prescription data from pharmacies. The Rotterdam study was approved by the medical ethics committee of the Erasmus Medical Center, Rotterdam, the Netherlands and by the review board of the Netherlands Ministry of Health, Welfare and Sports (1068889-159521-PG). All participants provided written informed consent.

\section{Study population}

All participants were included in this study and consisted of respondents to the first questionnaires on chronic cough during the home interview performed between 2002 and 2008; in particular RS-I-4, RS-II-2, and RS-III-1. Follow-up time was defined as the time period between this round's home interview and the subsequent second home interview.

\section{Identification of participants with chronic cough}

Chronic cough was assessed by questionnaire and defined, in agreement with international definitions, as cough lasting for a continuous period of 3 months or more [6]. Participants were asked the following - "In the last 2 years, did you cough nearly daily for three consecutive months or more?" [19]. Those who answered "no" were identified as subjects without chronic cough while participants who answered "yes" were considered to have chronic cough at baseline. The incidence of chronic cough was investigated by following participants: free from chronic cough at baseline until the second home interview (questionnaire). Incident cases were defined as subjects without chronic cough at baseline who developed chronic cough during follow-up (as evidenced at the second interview).

\section{Covariables}

Covariables were assessed at baseline. Smoking status was assessed using questionnaire data. Body mass index (BMI) was calculated as the ratio of weight in kilogram to height in squared metres. Obesity was 
defined as a BMI $\geqslant 30 \mathrm{~kg} \cdot \mathrm{m}^{-2}$. Age was categorised into decades and smoking status reported as current, past and never smokers. Use of ACE inhibitors was determined using questionnaire data [20]. COPD cases were identified using spirometry data and medical records [21]. Asthma cases were validated based on medical records [22]. Participants who had both asthma and COPD at baseline were classified as having asthma-COPD overlap (ACO). Chronic rhinosinusitis and GORD were identified using pharmacy data. We reviewed the number of prescriptions a participant received within 1 year prior to baseline. Subjects who got at least two prescriptions of nasal steroids (Anatomical Therapeutic Chemical code (ATC) R01AD), within 1 year prior to baseline, were considered to have chronic rhinosinusitis. GORD was also defined as having more than two prescriptions of medications for acid-related disorders such as peptic ulcer or reflux disease (ATC A02B) within 1 year prior to baseline.

\section{Statistical analyses}

The baseline characteristics of the study population were presented using descriptive statistics and compared using the Mann-Whitney test for skewed continuous variables, Chi-squared test for categorical variables and t-test for normally distributed continuous data. The prevalence of chronic cough was calculated as the proportion of patients with chronic cough at baseline expressed in percentages with corresponding 95\% confidence intervals. The prevalence of chronic cough was reported, categorised by age, sex, smoking status, obesity and comorbidities (ACO, COPD, asthma, GORD and chronic sinusitis). The prevalence of chronic cough in these categories were compared using Chi-squared tests. To determine the number of subjects at risk of incident chronic cough, participants with prevalent chronic cough at baseline were excluded. Incidence rates of chronic cough were calculated by dividing the number of incident cases by the total number of person years of subjects at risk and reported per 1000 person years. The $95 \%$ confidence intervals of the incidence rates were calculated using a Poisson distribution. Incidence rates were reported for age, sex, smoking status, obesity and comorbidities. The incidence rate of chronic cough between groups were compared with incidence rate ratio (IRR) estimated by Poisson regression. We calculated the incidence rate of chronic cough in participants with complete follow up and also in all the participants eligible for follow up, as a sensitivity analysis. For the sensitivity analysis, we defined follow up as the time period between the first round of home interview and death, date of last contact with the research centre or the end of the study (June 31, 2014) whichever came first. There were no acute calendar dates of onset of chronic cough; therefore, we performed logistic regression (instead of Cox regression) to determine the risk factors of chronic cough and adjusted for sex, smoking, comorbidities and potential confounders. Statistical significance was considered at a p-value of $<0.05$. Statistical analyses were performed using SPSS statistical software (SPSS for Windows, version 24; SPSS; Chicago, IL, USA) and R-software (The R Project for Statistical computing; www.r-project.org).

\section{Results}

\section{Baseline characteristics}

Of the 14926 subjects in the Rotterdam Study, 2998 persons (20.0\%) died and 28 individuals $(<1 \%)$ left the study before the first interview on chronic cough. About $80 \%$ of the total cohort $(n=11900)$ were invited to participate in the first interview; 9984 subjects out of 11900 invitees actually participated, representing a participation rate of $83.9 \%$. Also, $98.4 \%(n=9824)$ of the participants responded to the chronic cough questionnaire administered during the first interview.

The baseline characteristics of the study population are shown in table 1 . Figure 1 depicts the STROBE (Strengthening the Reporting of Observational Studies in Epidemiology) study flow chart. The mean \pm SD age of the study population was $66.0 \pm 10.8$ years and $58.3 \%(n=5725)$ of them were women. Subjects with baseline chronic cough were more frequent in the eighth decade and above $(\mathrm{p}<0.001)$ and had significantly more often a history of current smoking $(\mathrm{p}<0.001)$, use of ACE inhibitors $(\mathrm{p}<0.001)$, and comorbidities $(p<0.001)$. There were 37737 years of observation for 6245 participants with complete follow-up. Of the 8751 participants without baseline chronic cough and who were eligible for follow-up, 1480 (16.9\%) participants died during follow-up and 1026 (11.7\%) participants were unavailable at the end of the follow-up period.

\section{Prevalence of chronic cough}

The overall baseline period prevalence of chronic cough in the cohort was $10.9 \%$ (95\% CI 10.3-11.6). The prevalence of chronic cough increased with age and peaked in the eighth decade (See fig. 2 and table 2). Although there were no significant sex-specific differences in the prevalence of chronic cough in the overall study population ( $\mathrm{p}=0.369$ ), in subjects younger than 70 years, the prevalence of chronic cough was significantly higher in women $(10.2 \%, 95 \%$ CI 9.2-11.3) than in men $(8.5 \%, 95 \%$ CI $7.4-9.6) ; \mathrm{p}=0.023$. Generally, the prevalence of chronic cough was significantly higher in current smokers $(18.6 \%, 95 \%$ CI 16.8-20.5) than in never smokers (8.6\%, 95\% CI 7.6-9.6), $\mathrm{p}<0.001$; and in ACE inhibitors users 


\begin{tabular}{|c|c|c|c|c|}
\hline Baseline characteristics & Total & Chronic cough cases & Non-cases & p-value \\
\hline Subjects $\mathbf{n}$ & 9824 & 1073 & 8751 & \\
\hline Age years & $66.0 \pm 10.8$ & $67.8 \pm 10.6$ & $65.8 \pm 10.8$ & 0.392 \\
\hline \multicolumn{5}{|l|}{ Age $\geqslant 70$ years } \\
\hline No & 6118 (62.3) & $579(54.0)$ & 5539 (63.3) & \multirow[t]{2}{*}{$<0.001$} \\
\hline Yes & 3706 (37.7) & $494(46.0)$ & 3212 (36.7) & \\
\hline \multicolumn{5}{|l|}{ Age (decades) } \\
\hline $5^{\text {th }}$ & $570(5.8)$ & $39(3.6)$ & $531(6.1)$ & \multirow[t]{5}{*}{$<0.001$} \\
\hline $6^{\text {th }}$ & $2297(23.4)$ & 209 (19.5) & $2088(23.9)$ & \\
\hline $7^{\text {th }}$ & $3251(33.1)$ & $331(30.8)$ & $2920(33.4)$ & \\
\hline $8^{\text {th }}$ & $2453(25.0)$ & 338 (31.5) & $2115(24.2)$ & \\
\hline$\geqslant 9^{\text {th }}$ & $1253(12.8)$ & $156(14.5)$ & 1097 (12.5) & \\
\hline Female sex & $5725(58.3)$ & $639(59.6)$ & $5086(58.1)$ & 0.369 \\
\hline \multicolumn{5}{|l|}{ Smoking } \\
\hline Never & 3133 (31.9) & $268(25.0)$ & 2865 (32.8) & \multirow[t]{3}{*}{$<0.001$} \\
\hline Past & $4915(50.0)$ & 475 (44.3) & $4440(50.8)$ & \\
\hline Current & $1769(18.0)$ & $329(30.7)$ & 1440 (16.5) & \\
\hline BMI $\mathrm{kg} \cdot \mathrm{m}^{-2}$ & $27.1(24.7-29.9)$ & $27.2(24.6-30.4)$ & $27.0(24.7-29.8)$ & 0.475 \\
\hline Obesity & $2114(21.5)$ & $240(22.4)$ & $1874(21.4)$ & 0.154 \\
\hline ACE inhibitors & $1837(18.7)$ & $244(22.7)$ & 1593 (18.2) & $<0.001$ \\
\hline Comorbidities & $2420(24.6)$ & $480(44.7)$ & $1940(22.2)$ & $<0.001$ \\
\hline Chronic rhinosinusitis & 289 (2.9) & $57(5.3)$ & $232(2.7)$ & $<0.001$ \\
\hline GORD & 1324 (13.5) & 196 (18.3) & $1128(12.9)$ & $<0.001$ \\
\hline Asthma & $522(5.3)$ & $124(11.6)$ & $389(4.5)$ & $<0.001$ \\
\hline COPD & $646(6.6)$ & 213 (19.9) & 433 (4.9) & $<0.001$ \\
\hline $\mathrm{ACO}$ & $21(0.2)$ & $6(0.6)$ & $15(0.2)$ & 0.009 \\
\hline
\end{tabular}

(13.3\%, 95\% CI 11.8-14.9) than in non-users (10.4\%, 95\% CI 9.7-11.1), $\mathrm{p}<0.001$; but not significantly different in past smokers $(9.7 \%, 95 \%$ CI $8.9-10.5)$ compared to never smokers $(8.6 \%, 95 \%$ CI $7.6-9.6)$, $\mathrm{p}=0.620$; or in obese subjects $(11.4 \%, 95 \%$ CI $10.0-12.8)$ compared to non-obese subjects $(10.3 \%, 95 \%$ CI 9.5-11.0), $\mathrm{p}=0.154$. The prevalence of chronic cough according to comorbidities were as follows: $14.8 \%$ (95\% CI 12.9-16.8) in GORD, $19.7 \%$ (95\% CI 15.3-24.8) in chronic rhinosinusitis, $23.8 \%$ (95\% CI 20.2-27.6) in asthma, 33.0\% (95\% CI 29.4-36.7) in COPD, and 28.6\% (95\% CI 11.3-52.2) in ACO.

\section{Incidence rate of chronic cough}

During a mean follow-up of 6 years, 439 incident cases of chronic cough occurred with an overall incidence rate of 11.6 per 1000 person-years (95\% CI 10.6-12.8). As highlighted in table 3 and figure 3, there were no significant age-specific (IRR 0.93 , 95\% CI $0.75-1.15$ ) or sex-specific (IRR $0.95,95 \%$ CI 0.79-1.16) differences in the incidence rate of chronic cough in the overall study population. The incidence rate of chronic cough was higher in obese subjects than in non-obese subjects (IRR 1.27, 95\% CI 1.02-1.58) and in current smokers than in never smokers (IRR 1.57, 95\% CI 1.20-2.07). Notably, in current smokers, the incidence of chronic cough was higher in men than in women (IRR 1.92, 95\% CI 1.25-2.96). The incidence rate of chronic cough according to comorbidities were as follows: 15.2 per 1000 person-years (95\% CI 11.9-19.2) in GORD, 15.1 per 1000 person-years (95\% CI 8.7-24.6) in chronic rhinosinusitis, 26.2 per 1000 person-years (95\% CI 19.5-34.5) in asthma, 23.9 per 1000 person-years (95\% CI 16.7-33.3) in COPD, and 33.9 per 1000 person-years (95\% CI 4.1-122.5) in ACO. The results of the sensitivity analysis followed a similar trend as the results in the participants with complete follow-up except that the incidence rate of chronic cough decreased with age (IRR 0.64, 95\% CI 0.52-0.79). Other details are shown in table 4.

\section{Risk factors for chronic cough}

As shown in table 5, The age- and sex-adjusted odds ratios for incident chronic cough was significant in current smokers (OR 1.63, 95\% CI 1.23-2.16), and in subjects with obesity (OR 1.30, 95\% CI 1.04-1.62), GERD (OR 1.42, 95\% CI 1.09-1.86), asthma (OR 2.73, 95\% CI 1.98-3.75), and COPD (OR 2.42, 95\% CI 1.66-3.53). Furthermore, all determinants (age, sex, smoking status, obesity, chronic rhinosinusitis, GERD, 


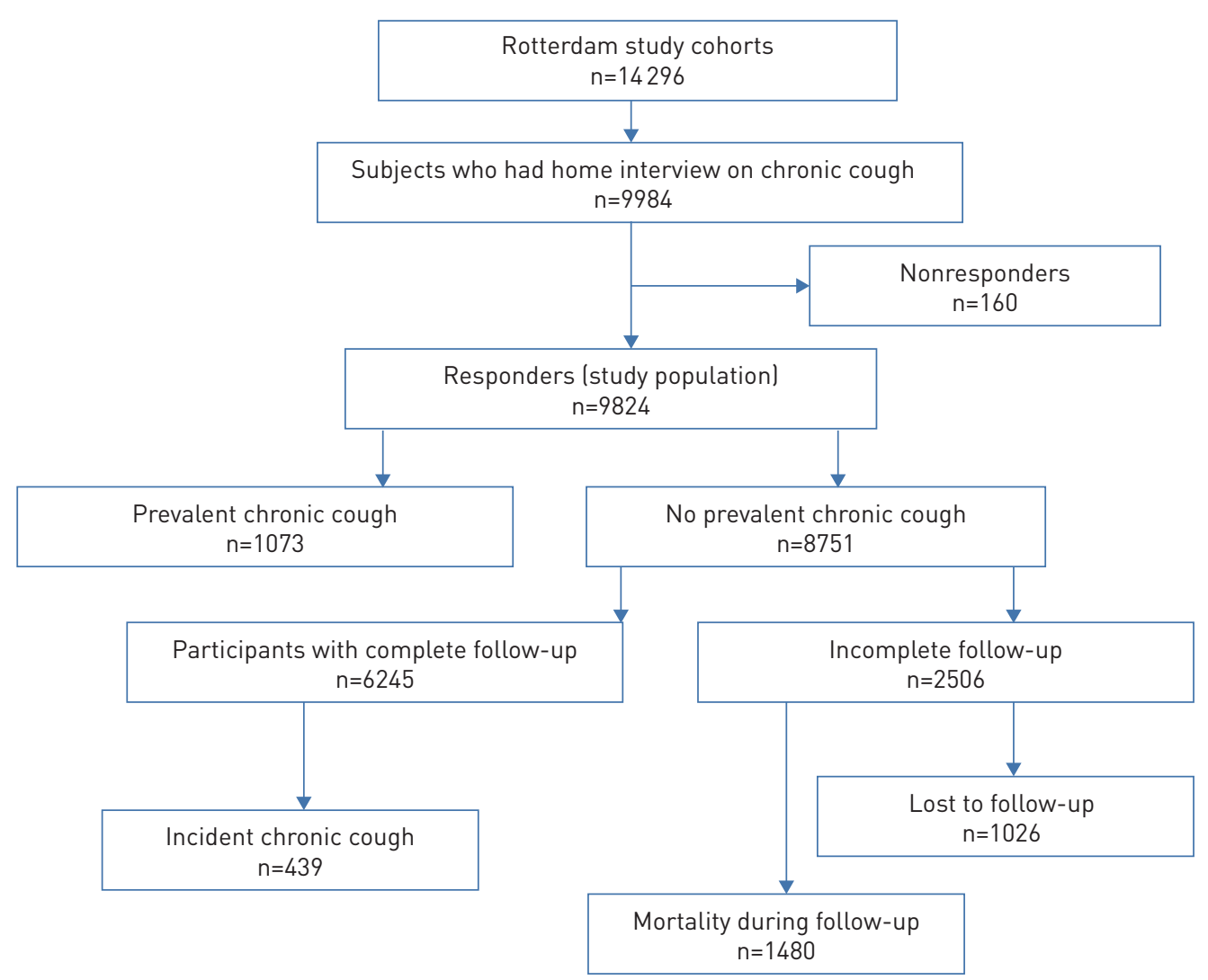

FIGURE 1 STROBE Study flow chart.

asthma, and COPD) were included in the multivariable model. The result showed that the risk of developing chronic cough was significantly increased in current smokers (OR 1.64, 95\% CI 1.22-2.19), and in subjects with GERD (OR 1.34, 95\% CI 1.01-1.78), asthma (OR 2.88, 95\% CI 2.07-4.02), and COPD (OR 2.52, 95\% CI 1.70-3.71). Moreover, the risk for chronic cough in subjects with obstructive airway disease (asthma, COPD, and ACO) was significantly increased (OR 2.75, 95\% CI 2.12-3.58).

\section{Discussion}

In this large prospective population-based cohort study, we investigated the prevalence and incidence of, and risks factors for chronic cough in a community-dwelling middle-aged and older population. Our results showed that the prevalence of chronic cough in the adult population is approximately $11 \%$ with an estimated incidence rate of 11.6 per 1000 person-years. In addition, current smoking, GORD, COPD and asthma were independent risk factors for chronic cough in our study population.

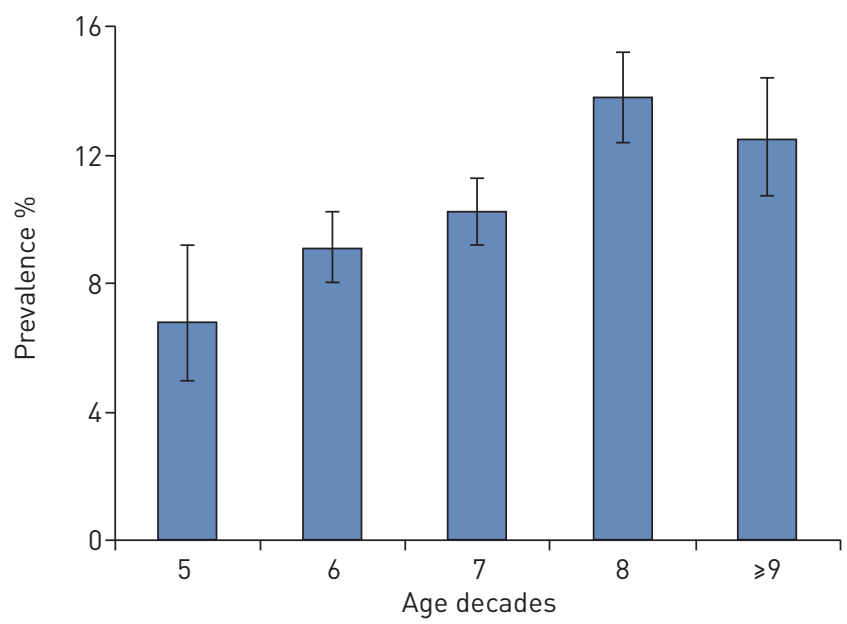




\begin{tabular}{|c|c|c|c|c|}
\hline Baseline characteristics & $\begin{array}{l}\text { Subjects at } \\
\text { risk } n\end{array}$ & $\begin{array}{l}\text { Prevalent chronic } \\
\text { cough } \mathrm{n}\end{array}$ & $\begin{array}{l}\text { Prevalence \% } \\
\text { (95\% CI) }\end{array}$ & p-value \\
\hline Study Population & 9824 & 1073 & $10.9(10.3-11.6)$ & \\
\hline \multicolumn{5}{|l|}{ Sex } \\
\hline Male & 4099 & 434 & $10.6(9.7-11.6)$ & \multirow[t]{2}{*}{0.369} \\
\hline Female & 5725 & 639 & $11.2(10.4-12.0)$ & \\
\hline \multicolumn{5}{|l|}{ Age $\geqslant 70$ years } \\
\hline No & 6118 & 579 & $9.5(8.7-10.2)$ & \multirow[t]{2}{*}{$<0.001$} \\
\hline Yes & 3706 & 494 & $13.3(12.3-14.5)$ & \\
\hline \multicolumn{5}{|l|}{ Age $<70$ years } \\
\hline Male & 2640 & 224 & $8.5(7.4-9.6)$ & \multirow[t]{2}{*}{0.023} \\
\hline Female & 3478 & 355 & $10.2(9.2-11.3)$ & \\
\hline \multicolumn{5}{|l|}{ Smoking status } \\
\hline Never & 3133 & 268 & $8.6(7.6-9.6)$ & \multirow[t]{3}{*}{$<0.001$} \\
\hline Past & 4915 & 475 & $9.7(8.9-10.5)$ & \\
\hline Current & 1769 & 329 & $18.6(16.8-20.5)$ & \\
\hline \multicolumn{5}{|l|}{ ACE-inhibitors } \\
\hline Non-users & 7987 & 829 & $10.4(9.7-11.1)$ & \multirow[t]{2}{*}{$<0.001$} \\
\hline Users & 1837 & 244 & $13.3(11.8-14.9)$ & \\
\hline \multicolumn{5}{|l|}{ Obesity } \\
\hline No & 6618 & 679 & $10.3(9.5-11.0)$ & \multirow[t]{2}{*}{0.154} \\
\hline Yes & 2114 & 240 & $11.4(10.0-12.8)$ & \\
\hline
\end{tabular}

The majority of patients seeking medical attention for chronic cough in out-patient clinics are adults [23]. We found that one in nine adults has chronic cough. Our finding is similar to the global prevalence of $10 \%$ and slightly lower than the estimated prevalence in Europe which is $12.7 \%$ [2]. ForD et al. [24] reported a prevalence of $12 \%$ in the adult UK population enrolled in a Helicobacter pylori screening and treatment programme. Moreover, the reported high prevalence of chronic cough in that study could be due to an expected higher prevalence of GORD in the selected population. Also, a lower prevalence of $4 \%$ was reported in 14669 Danish subjects [25]. However, in that population, subjects had a lower median age (58 years) than in our study population (mean age 66 years). In Finland, the point prevalence of daily chronic cough was $7.2 \%$ [26]. The study included a defined population of relatively young (baseline age $50.5 \pm 10.1$ years) Finnish adult employees with a low prevalence of smoking (ever smokers $-30.7 \%)$. In

\section{TABLE 3 Incidence rate of chronic cough in participants with complete follow up}

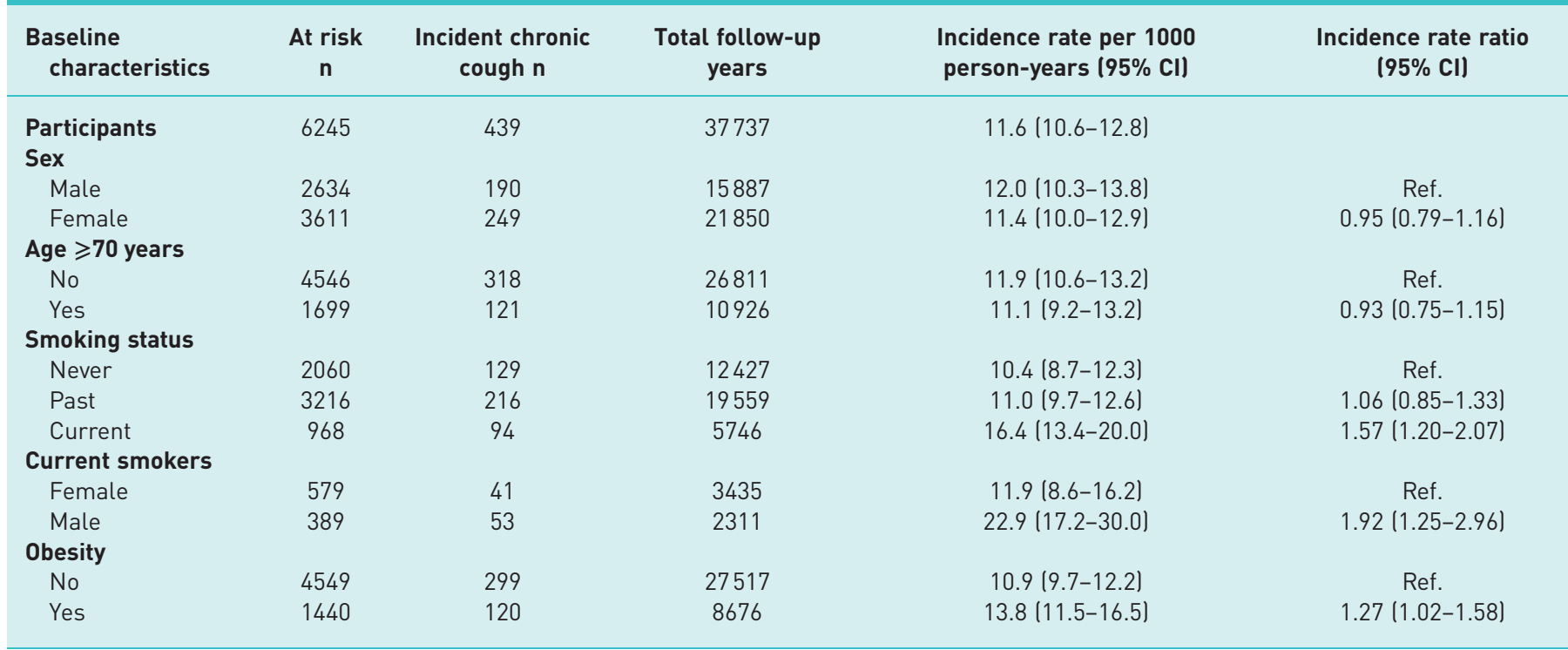


FIGURE 3 Age-specific incidence of chronic cough. PY: person-years.

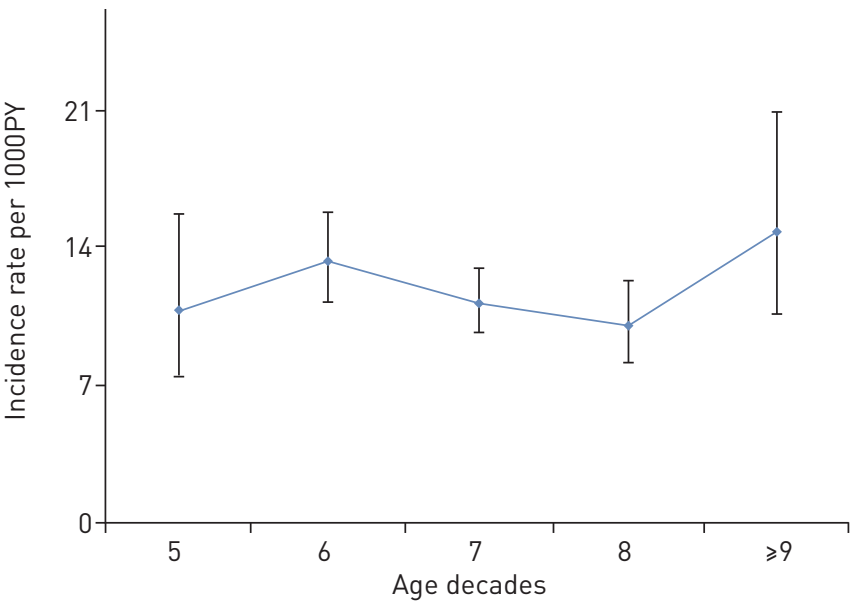

contrast, our study consisted of older subjects (baseline age $66.0 \pm 10.8$ years) from the general population with a high prevalence of smoking (ever smokers: $68.0 \%$ ).

Chronic cough is preponderant in females and the cough reflex is more sensitive in women [27]. We found that chronic cough was more prevalent in women in subjects below 70 years of age. Nevertheless, there were no significant sex-specific differences in the prevalence of chronic cough in the overall study population. Similarly, Song et al. [6] found no significant differences in the sex-specific prevalence of chronic cough. We observed that the prevalence of chronic cough increased with age and that chronic cough was more prevalent in subjects aged 70 years and above. Likewise, the results of the Korean National Health and Nutrition Examination Survey showed that chronic cough was more prevalent in elderly subjects [28].

The risk factors for chronic cough in adults have been reported in the medical literature [2]. However, most of these studies were mainly cross-sectional surveys and thus, gave limited insight into the aetiology of chronic cough. The prospective design of our study enabled us to reliably estimate the incidence of, and the risk factors for, chronic cough. Our study showed that about 12 out of 1000 adults develop chronic cough annually. Although the risk of smoking-related morbidity and mortality is known to be higher in women [29], we found that, in current smokers, the incidence of chronic cough was higher in men. This is plausible considering that current smoking, a strong independent risk factor for chronic cough, is more prevalent in men $[6,30]$.

\section{TABLE 4 Incidence rate of chronic cough in all eligible participants}

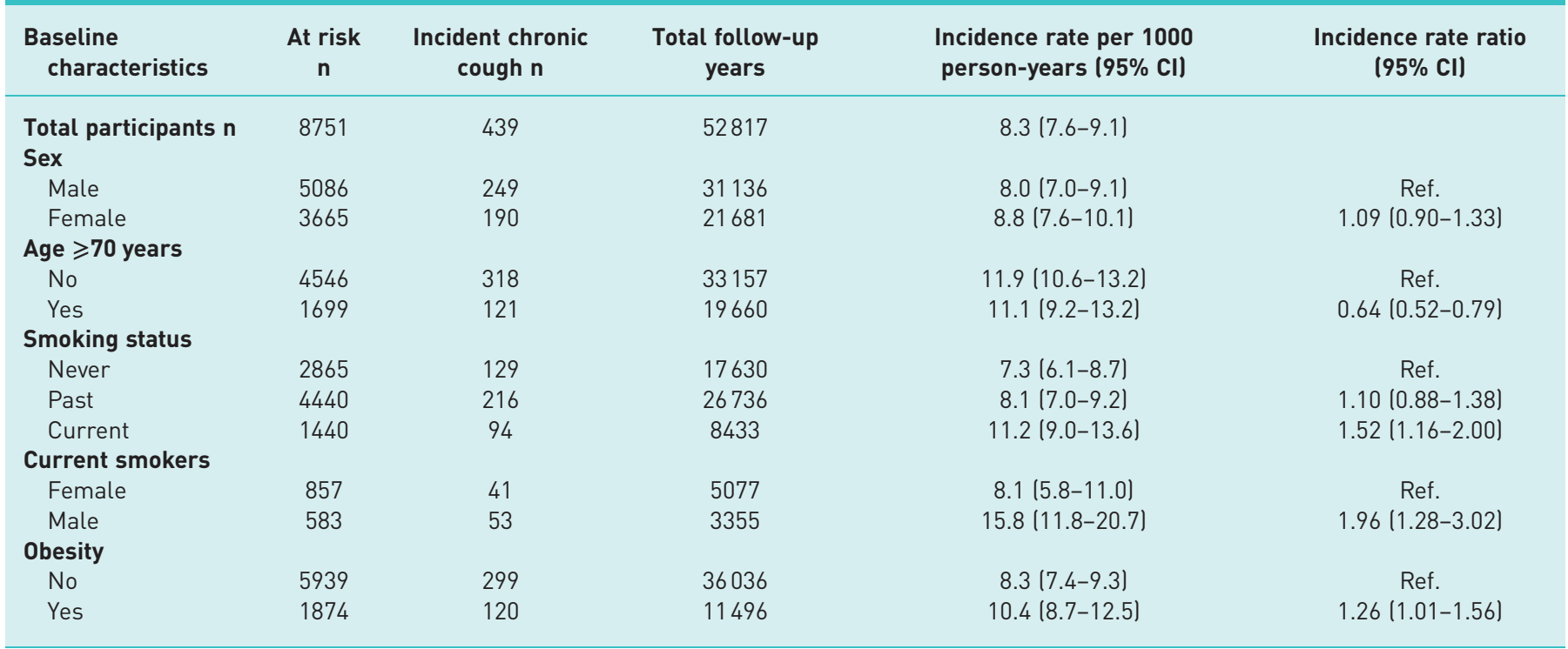




\section{TABLE 5 Risk factors for chronic cough}

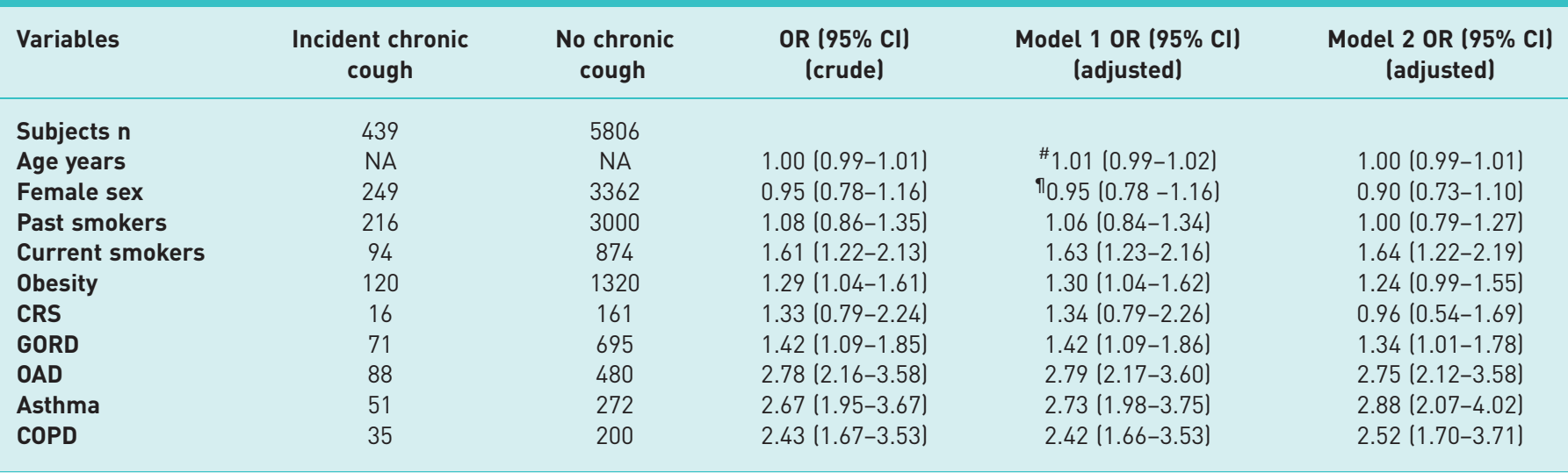

Model 1: adjusted for age and sex; model 2 (full model): adjusted for age, sex, smoking, obesity, chronic obstructive pulmonary disease (COPD), asthma, gastro-oesophageal reflux disease (GORD) and chronic rhinosinusitis (CRS). Asthma-COPD overlap (ACO) was omitted from the analyses due to low power (10 subjects). OAD: obstructive airway disease (asthma, COPD and ACO). \#: adjusted for sex and smoking. १: adjusted for age and smoking.

Furthermore, current smoking, GORD, asthma and COPD were independent risk factors for chronic cough in our study population. Our findings are in line with the results of previous epidemiological studies on chronic cough $[25,31]$. Smoking is an important risk factor for chronic cough and current smokers are known to have an increased cough frequency [32, 33]. Also, GORD has been reported as the third most important medical condition associated with chronic cough in Europe [11]. More still, chronic cough is not only common but also associated with a more severe clinical outcome in patients with obstructive airway disease $[34,36]$. In our study, chronic cough was most prevalent in COPD patients and new cases of chronic cough occurred most in subjects with asthma. On the whole, obstructive airway disease was the most important risk factor for chronic cough in our study population.

The strength of the Rotterdam Study is its prospective, population-based design with long follow-up time and similar data collection procedures for every subject. Measurement of variables were independent of the research question thereby making them less susceptible to selection and information bias. In addition, the effect of selection bias is less likely due to the high response rate $(98 \%)$ to the questionnaire on chronic cough.

Like most observational studies, this study is not without potential limitations. First, the Rotterdam Study adopted a duration of 3 months and a two-year period for defining chronic cough; which was the most commonly used epidemiological definition at the time of data collection [2]. Nevertheless, it differs from the criterion of current clinical guidelines (duration of $\geqslant 8$ weeks); moreover, the two-year period is susceptible to recall bias. However, the meta-analysis by Song et al. [6] showed that the prevalence data from studies using either the 8 weeks' or 3 months' duration, to define chronic cough, fell within the regional estimates. Importantly, our estimated prevalence of chronic cough (10.9\%) is consistent with the reported pooled prevalence of chronic cough in Europe (12.7\% (95\% CI 10.4-15.2\%)) [2]. Therefore, the effect of this limitation is expected to be minimal. Secondly, it was not possible for us to report the point prevalence of chronic cough; hence, we estimated a period prevalence that covered any episode of chronic cough that occurred within the previous 24 months before the time of the survey. Thirdly, we had a considerable attrition rate (28.6\%), due to mortality and lost to follow-up, and this could have led to underestimation of the incidence rate and risk of chronic cough in this population. However, we used person-years rather than persons as denominator to ensure an accurate estimation of the incidence (rate) of chronic cough. Also, the results of our sensitivity analysis including all participants eligible for follow up had a similar trend with the findings in subjects with complete follow-up. Therefore, the impact of the attrition on the estimation of incidence and risk of chronic cough is expected to be minimal. Fourthly, due to lack of data on chronic rhinosinusitis and GERD, we used medication for these indications as a proxy to identify subjects with these medical conditions. This may have resulted in misclassification of disease as subjects with fewer prescriptions may be under-diagnosed and those with more prescriptions over-diagnosed. Fifthly, incident chronic cough was assessed after 6 years (mean duration) of follow-up and we did not have information on the exact date of first diagnosis of chronic cough. Hence, the person-years from baseline to second interview date was used and this may have led to a slight underestimation of incidence rate of chronic cough in this population. 
In conclusion, chronic cough is common among adults and highly prevalent in the older population. Smoking, GORD, and obstructive airway disease are independent risk factors of chronic cough. Individuals at risk of developing chronic cough may benefit from smoking cessation and control of underlying diseases.

Conflict of interest: J.T. Arinze has nothing to disclose. E.W. de Roos has nothing to disclose. L. Karimi has nothing to disclose. K.M.C. Verhamme works for a research group that, in the past, received unconditional grants from Yamanouchi, Pfizer/Boehringer Ingelheim, Novartis and GSK, none of which relate to the content of this work. B.H. Stricker has nothing to disclose. G.G. Brusselle reports advisory boards and speaker's fees from AstraZeneca, Boehringer Ingelheim, Chiesi, GlaxoSmithKline, Novartis and Teva; and advisory board fees from Sanofi, outside the submitted work.

\section{References}

1 Schappert SM, Nelson C. National Ambulatory Medical Care Survey: 1995-96 summary. Vital Health Stat 13 1999; 142: 1-122.

2 Song WJ, Chang YS, Faruqi S, et al. The global epidemiology of chronic cough in adults: a systematic review and meta-analysis. Eur Respir J 2015; 45: 1479-1481.

3 Koskela HO, Lätti AM, Purokivi MK. Long-term prognosis of chronic cough: a prospective, observational cohort study. BMC Pulm Med 2017; 17: 146-146.

4 Morice AH, Millqvist E, Bieksiene $\mathrm{K}$, et al. ERS guidelines on the diagnosis and treatment of chronic cough in adults and children. Eur Respir J 2020; 55: 1901136.

5 Irwin RS, French CL, Chang AB, et al. Classification of cough as a symptom in adults and management algorithms: CHEST guideline and expert panel report. Chest 2018; 153: 196-209.

6 Song WJ, Chang YS, Faruqi S, et al. Defining chronic cough: a systematic review of the epidemiological literature Allergy Asthma Immunol Res 2016; 8: 146-155.

$7 \quad$ Smith JA, Woodcock A. Chronic cough. N Engl J Med 2016; 375: 1544-1551.

8 Tarlo SM, Altman KW, Oppenheimer J, et al. Occupational and environmental contributions to chronic cough in adults: chest expert panel report. Chest 2016; 150: 894-907.

9 Pratter MR. Overview of common causes of chronic cough: ACCP evidence-based clinical practice guidelines Chest 2006; 129: 59S-62S.

10 Haque RA, Usmani OS, Barnes PJ. Chronic idiopathic cough: a discrete clinical entity? Chest 2005; 127: $1710-1713$.

11 Chamberlain SA, Garrod R, Douiri A, et al. The impact of chronic cough: a cross-sectional European survey. Lung 2015; 193: 401-408.

12 Morice AH. Chronic cough hypersensitivity syndrome. Cough 2013; 9: 14

13 Escamilla R, Roche N. Cough hypersensitivity syndrome: towards a new approach to chronic cough. Eur Respir J 2014; 44: 1103-1106.

14 Dicpinigaitis PV, Kantar A, Enilari O, et al. Prevalence of Arnold nerve reflex in adults and children with chronic cough. Chest 2018; 153: 675-679.

15 Song WJ, Morice AH, Kim MH, et al. Cough in the elderly population: relationships with multiple comorbidity. PLoS One 2013; 8: e78081.

16 Morice AH, Jakes AD, Faruqi S, et al. A worldwide survey of chronic cough: a manifestation of enhanced somatosensory response. Eur Respir J 2014; 44: 1149-1155.

17 Hofman A, Brusselle GG, Darwish Murad S, et al. The Rotterdam Study: 2016 objectives and design update. Eur J Epidemiol 2015; 30: 661-708.

18 Ikram MA, Brusselle GGO, Murad SD, et al. The Rotterdam Study: 2018 update on objectives, design and main results. Eur J Epidemiol 2017; 32: 807-850.

19 Vestbo J, Hurd SS, Agusti AG, et al. Global strategy for the diagnosis, management, and prevention of chronic obstructive pulmonary disease: GOLD executive summary. Am J Respir Crit Care Med 2013; 187: $347-365$.

20 Dicpinigaitis PV. Angiotensin-converting enzyme inhibitor-induced cough: ACCP evidence-based clinical practice guidelines. Chest 2006; 129: Suppl. 1, 169S-173S.

21 van Durme YMTA, Verhamme KMC, Stijnen T, et al. Prevalence, incidence, and lifetime risk for the development of COPD in the elderly: The Rotterdam Study. Chest 2009; 135: 368-377.

22 de Roos EW, Lahousse L, Verhamme KMC, et al. Asthma and its comorbidities in middle-aged and older adults; the Rotterdam Study. Respir Med 2018; 139: 6-12.

23 Dicpinigaitis PV. Thoughts on one thousand chronic cough patients. Lung 2012; 190: 593-596.

24 Ford AC, Forman D, Moayyedi P, et al. Cough in the community: a cross sectional survey and the relationship to gastrointestinal symptoms. Thorax 2006; 61: 975-979.

25 Colak Y, Nordestgaard BG, Laursen LC, et al. Risk factors for chronic cough among 14,669 individuals from the general population. Chest 2017; 152: 563-573.

26 Lätti AM, Pekkanen J, Koskela HO. Defining the risk factors for acute, subacute and chronic cough: a cross-sectional study in a Finnish adult employee population. BMJ Open 2018; 8: e022950.

27 Kastelik JA, Thompson RH, Aziz I, et al. Sex-related Differences in Cough Reflex Sensitivity in Patients with Chronic Cough. Am J Respir Crit Care Med 2002; 166: 961-964.

28 Kang M-G, Song W-J, Kim H-J, et al. Point prevalence and epidemiological characteristics of chronic cough in the general adult population: The Korean National Health and Nutrition Examination Survey 2010-2012. Medicine 2017; 96: e6486-e6486.

29 Allen AM, Oncken C, Hatsukami D. Women and smoking: the effect of gender on the epidemiology, health effects, and cessation of smoking. Curr Addict Rep 2014; 1: 53-60.

30 Allen AM, Scheuermann TS, Nollen N, et al. Gender differences in smoking behavior and dependence motives among daily and nondaily smokers. Nicotine Tob Res 2016; 18: 1408-1413.

31 Koo H-K, Jeong I, Lee SW, et al. Prevalence of chronic cough and possible causes in the general population based on the Korean National Health and Nutrition Examination Survey. Medicine 2016; 95: e4595-e4595. 
32 Kanezaki M, Ebihara S, Gui P, et al. Effect of cigarette smoking on cough reflex induced by TRPV1 and TRPA1 stimulations. Respir Med 2012; 106: 406-412.

33 Sumner $\mathrm{H}$, Woodcock A, Kolsum U, et al. Predictors of objective cough frequency in chronic obstructive pulmonary disease. Am J Respir Crit Care Med 2013; 187: 943-949.

34 Koo H-K, Park S-W, Park J-W, et al. Chronic cough as a novel phenotype of chronic obstructive pulmonary disease. Int J Chron Obstruct Pulmon Dis 2018; 13: 1793-1801.

35 Lahousse L, Seys LJM, Joos GF, et al. Epidemiology and impact of chronic bronchitis in chronic obstructive pulmonary disease. Eur Respir J 2017; 50: 1602470.

36 Çolak Y, Afzal S, Lange P, et al. Role and impact of chronic cough in individuals with asthma from the general population. J Allergy Clin Immunol Pract 2019; 7: 1783-1792. 